\title{
Yeast as Carrier for Drug Delivery System
}

\author{
Anil Gutte
}

\begin{abstract}
:
The microbes are used for the treatment of clinical conditions for historical technology, use of microbes provide many advantages of microbes to human. Their potential within the prevention and remedy of a wide range of disorder situations has been appeared for the cause that days of Jenner, who in 1796 inoculated a younger boy with cowpox and later with smallpox. the usage of modified non-virulent species, attenuated bacteria/viruses and purified parts of micro-organisms has made vaccination one of the finest achievements of drugs, and has spared tens of thousands and thousands of people from the outcomes of devastating ailments. similarly, the use of microbes as mini-mobile factories for the manufacturing of antibiotics, vaccines, biopharmaceuticals and some of essential excipients (as an instance, non-animal derived albumin) has contributed broadly to improvements in health care. in this evaluation we can study the microencapsulation of drug in yeast cells for education of oral, topical and targeted drug delivery machine
\end{abstract}

Key Words: Microbes, Treatment, Microencapsulation, Oral Drug Delivery, Targeted Drug Delivery etc.

Introduction: Current advances have suggested the use of nonpathogenic microorganism - together with Lactobacillus traces, that are usually found in organic yogurt - for less steeply-priced mucosalprimarily based vaccines. sorts of live / baker yeast have additionally been shown to play a function, and enteric-primarily based products are already available on the market in fitness meals shops - and in a few countries on prescription - to treat situations including ulcerative colitis and irritable bowel syndrome (IBS). to test other applications, microbes - and particularly yeast cells - had been proposed in the early Seventies by way of Joseph Shank, who works for rapid and Co, as microcapsules are preprogrammed to combine a huge variety of active substances. His paintings, and later that of Nahida Pannell of AD2 Ltd (Birmingham, uk), targeted on the commercial use of yeast cells, for instance, in carbonloose replica paper and the transport of flavors. flavor transport technology is advancing and has now been delivered lower back to market via a partnership among Micap percent (Merseyside, uk) and Firmenich SA, a Swiss agency for flavor and aroma. ${ }^{1-5}$ Microencapsulation inside a herbal capsule with partitions of yeast cells allows for a bendy flavor to be 
processed and cooked, in the end bringing the flavor wherein it is wished inside the mouth. Examples of powerful applications encompass more desirable flavors in fried coatings, soups and sauces. This primary venture makes a speciality of using yeast-based totally microencapsulation generation as a brand new way to convert fluid into powder for easy handling. when it become realized that yeast tablets release their paid flavor whilst they come in touch with oral mucosal greater without problems than saliva on my own, the possibility of focused drug shipping the use of yeast cellular tablets became first proposed. while found inside the tongue, yeast cells are discovered to be strong after powerful delivery of taste. The era has in view that been progressed in oral transport, and is capable of reaching an active element load of up to $40 \%$ by using weight of lipophilic liquid or a portion of melted lipophilic in a suitable carrier. ${ }^{5-8}$

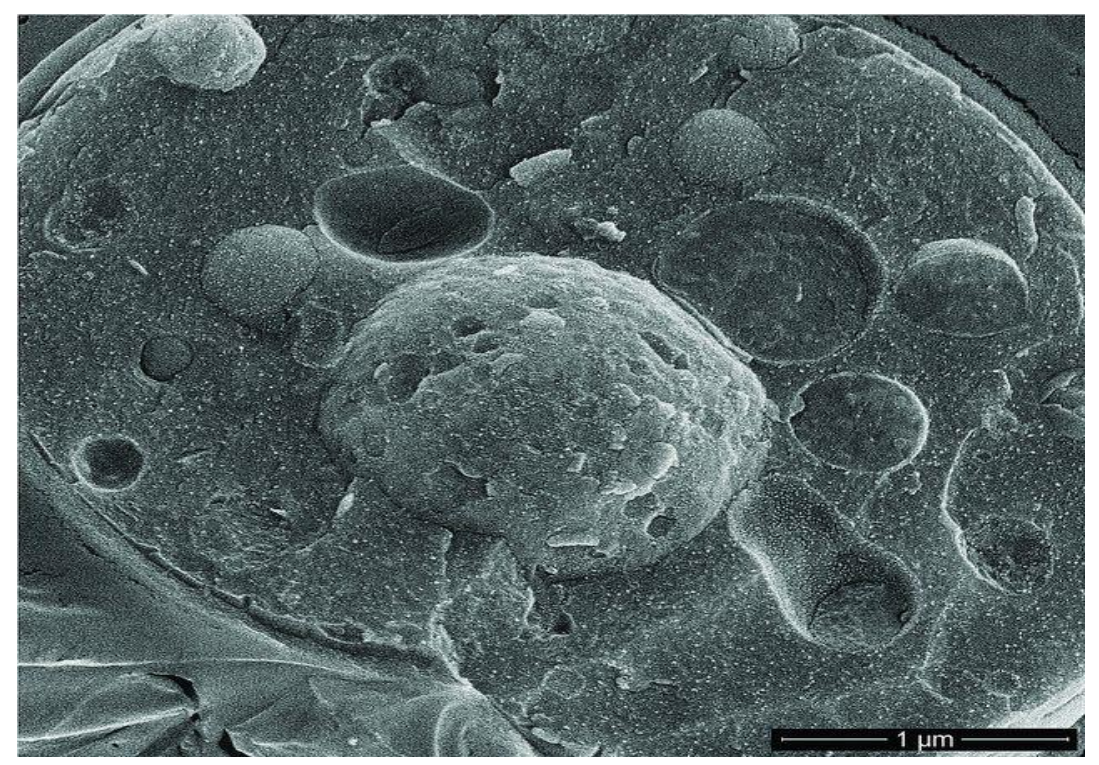

Fig 1: SEM image of Yeast cell with microencapsulated API

\section{Yeast as a Raw Material:}

Yeast has been planted through man for use as a brewery and wine maker and for bread for hundreds of years. it's

miles now available in large portions produced particularly through afew ingredients suppliers international consisting of DSM, Lallem and and Lesaffre. another important supply of yeast is provided with the aid of many US and South American producers of biofuel (ethanol), in which the enormous availability of sugar crops, as well as authorities subsidies, makes the production of ethanol from ripe yeast to be introduced to motor gasoline a possible proposal. ${ }^{9,10,11}$ 
Yeast is a molecular fungus and, even though there are numerous species that reproduce by means of germination or differentiation, the maximum commercially crucial is the Saccharomyces, which may also be the most commonplace form of yeast. Ovoid prepared, the average Saccharomyces cell is set five microns extensive, compared to other forms of yeast that may be as big as 20 microns wide. The yeast shape is defined by way

of a cell wall physically sturdy shape whose characteristic is primarily to guard the inner layer of yeast and organelles from the environment (Figure 1). ${ }^{12-15}$

The cellular wall is made from complex and fantastically glued glucan, mannan and, to a lesser quantity, chitin is related to scars left after the daughter cell has ruptured within the mother. The wall is ready 100$200 \mathrm{~nm}$ thick and covers $15-25 \%$ of the dry cellular weight. The cell wall surrounds a totally thin plasma membrane (<10 micron) - a standard blister unit inclusive of phospholipids, sterols and impartial lipids, represented particularly by using triacyl glycerol and sterol esters. currently, Saccharomyces' yeast types are produced in step with the dietary stage of beer, wine, drinks and bread; a few species are also produced at nutraceutical tiers. not like the newly advanced polymer transport case, the protection of yeast for oral and topical use (yeast is widely used in cosmetic production) is properly installed; this need to lead to easier adoption of drug delivery system. Yeast is currently utilized in cGMP-based totally methods for the production of vaccines and biopharmaceuticals, and the cGMP yeast for the mixing and delivery of lively pharmaceutical elements might be to be had soon. primarily based on characteristic with targeted excretion inside the mucosal subcutaneous areas, it has been cautioned that it is possible that different biological factors may promote the release of lively drugs. even though the discharge has nolonger but been achieved via epidermal pores and skin layers, it's been observed that contact with different dwelling organisms can result in release. ${ }^{16-20}$

\section{Microencapsulation procedure:}

Lipophilic energetic ingredients are divided into yeast mobile - a process driven by using the lipid bilayer membrane of a cellular. even though the stabilization mechanism has now not yet been completely decided, lipid-derived substances from mobile membranes and organelles seem to undergo conformational adjustments, form micellular systems within the cell, and the active factor is held as many drops about $20 \mathrm{~nm}$ extensive. ${ }^{21}$

Typically, the manner includes the preparation of yeast-slurry in water; the slurry is stirred the use of a top- 
paddle stirrer and an lively liquid factor is introduced. The

stirrer velocity is cautiously managed to determine the precise droplet length for proper dispersion, and the vessel is heated to 400C; this lightly melts the

yeast mobile membrane, without damaging its shape, allowing the entry of the energetic ingredient at an active rate. within 1-four hours, greater than eighty five\% of the energetic element will be accumulated internal yeast cellular tablets. ${ }^{22,23}$ The yeast mobile wall acts as a molecular filter, in order that only molecules with a small footprint of cells can unfold to the cellular. normally, which means molecules weighing less than 1,000 dalton cells can be used. on the stop of the encapsulation process, inactive yeast cells are harvested by way of centrifugation, rinsed with water and if vital dried by spray, fluidized-bed or ice-drying methods. a typical spray-dried product will encompass agglomerates of many yeast cells with a diameter of 30 microns. ${ }^{24}$ The easily dispersed agglomerates descend right into a single cell of approximately 5 microns wide while uncovered to water. Instead, dry powder can be formulated into tablets or capsules. ${ }^{25}$

\section{Used To prepare Following Drug delivery system:}

\section{Oral Delivery:}

The yeast microencapsulation machine is designed to incorporate high concentrations of energetic lipophilic compounds, and is capable of include an energetic compound into the mucosal selective website. Coating the yeast particles with not unusual substances (e.g., enteric coating structures) lets in centered transport of yeast and subsequent shipping of lively lipophilic everywhere from the mouth to the decrease gut. as soon as released, the yeast cells disperse inside the fluid of the GI tract and, in the mucosal area, supply their contents for incorporation into the circulatory device. In this example, a not unusual instance is proven through the anticholesterol agent, fenofibrate; while introduced into the duodenum, it well-knownshows a 'low burst' impact and allows the lengthy-time period transport of an energetic factor leading to a totally high degree of bioavailability. ${ }^{26,27}$

\section{Topical Drug Delivery:}

based totally on characteristic with centered launch in the mucosal subcutaneous regions, it's been recommended that it is feasible that different biological factors may also sell the discharge of energetic pills. although the discharge has not but been performed via epidermal pores and skin layers, it's been observed that contact with other living organisms can result 
in launch. especially sufferers who've been correctly killed by means of yeast-dealt with antibiotics encompass Candida albicans (typically called thrush) and methaphillin-resistant Staphylococcus aureus (MRSA) which, in the laboratory, all of the known epidemic strains have been correctly neutralised. In a evaluation with an antifungal topical cream already available on the market, it became determined that a yeast- microencapsulated formula containing the identical attention of lively component become as a minimum 5 instances more powerful, and that the pace of kill changed into additionally substantially superior (parent four). The yeast microcapsules may be incorporated into an expansion of traditional cream bases and may be changed to form an aqueousbased cream without the addition of different excipients. ${ }^{28,29}$

\section{Used For Taste Masking:}

The yeast-primarily based microencapsulation generation turned into in the beginning developed for products including unstable lipophilic flavours. Sensory evaluation of encapsulated flavours confirmed that, as compared with non-encapsulated flavours, the encapsulated product gave both an greater flavour (usually extra intensity) and that flavour notion became extended. The improved flavour transport homes, and possibilities for the focused on of flavour and drug launch, can be due at least partly to the traits of the yeast mobile-floor. this is, universal, a negatively charged cloth mainly because of the presence of phosphomannans and frequently possesses protruding nodules (bud scars). Combining the powerful delivery of flavour with the shipping of medicine can produce an effective flavor-covering solution. With cautious preference of flavour and manipulation of lipophilicity in comparison with the lively substance, it's far feasible to manipulate the discharge of the flavour inside the mouth, at the same time as maintaining the drug inside the yeast pill. it's also viable to permit both flavour and drug to launch collectively In either case, because of the power of flavour transport, simplest the quality taste is perceived. because the yeast microencapsulation gadget retains the energetic factor inside the yeast mobile tablet in water and other aqueous combinations, it's far feasible to provide not simplest a dry powder or tableted formulation for flavor-covering, however also a syrup or comparable water-based totally system. on this form, the product may be mainly suitable for paediatric sufferers. ${ }^{29-31}$

Conclusion: The general public are much less conscious that germsexist without being infected. micro organism - 'insects' - are in all likelihood regarded as undesirable due to their position in sickness or food spoilage. yet over the last two hundred years, and in particular for the 
reason that 1940's, germs have played a important position in enhancing human health.

The improvement of microencapsulation the use of yeast and / or bacteria as pre-

targeted drug transport automobiles will preserve this procedure of development. possibilities exist nowada ys for oral, topical and hidden flavors the use of yeast microencapsulation generation. in addition, because of the potential to trade the chemical composition of yeast cellular and membrane walls (the usage of considerable genetic records and yeast formation), extra targeted transport may additionally become a fact in the future.

\section{Reference:}

1. E. Semo, E. Kesselman, D. Danino, Y.D. Livney, Food Hydrocoll. 21(5), 936-942 (2007)

2. M. Eslami, M. Shahedi, M. Fathi, Food Biophys. 1-8 (2018)

3. E.I. Paramera, S.J. Konteles, V.T. Karathanos, Food Chem. 125(3), 892-902 (2011)

4. G. Shi, L. Rao, H. Yu, H. Xiang, G. Pen, S. Long, C. Yang, J. Food Eng. 80(4), 1060-1067 (2007)

5. S. Blanquet, S. Marol-Bonnin, E. Beyssac, D. Pompon, M. Renaud, M. Alric, Trends Biotechnol. 19(10), 393-400 (2001)

6. S. Blanquet, G. Garrait, E. Beyssac, C. Perrier, S. Denis, G. Hébrard, M. Alric, Eur. J. Pharm. Biopharm. 61(1), 32-39 (2005)

7. M. Kavosi, A. Mohammadi, S. Shojaee-Aliabadi, R. Khaksar, S.M. Hosseini, J. Sci. Food Agric. 98(7), 2490-2497 (2017)

8. Y. Luo, Z. Teng, Q. Wang, J Agric Food Chem 60(3), 836-843 (2012)

9. A. da Silva Lima, A.P. Maciel, C.D.J.S. Mendonça, L.M. Costa Junior, Ind. Crop. Prod. 108, 190194 (2017)

10. A. Sultana, A. Miyamoto, Q.L. Hy, Y. Tanaka, Y. Fushimi, H. Yoshii, J. Food Eng. 199, 36-41 (2017).

11. G. Tian, Y. Li, Q. Yuan, L. Cheng, P. Kuang, P. Tang, Carbohydr. Polym. 122, 5-10 (2015).

12. Y. Lin, Y.-H. Wang, X.-Q. Yang, J. Guo, J.-M. Wang, LWT - Food Sci Technol. 72, 510-517 (2016).

13. U.A. Guler, M. Sarioglu, Desalin. Water Treat. 52(25-27), 4871-4888 (2014).

14. M. Pantić, Ž. Knez, Z. Novak, J. Non-Cryst. Solids 432, 519-526 (2016).

15. M. Cavagna, R. Dell'Anna, F. Monti, F. Rossi, S. Torriani, J Agric Food Chem 58(1), 39-45 (2010).

16. Z. Teng, Y. Luo, Q. Wang, Food Chem. 141(1), 524-532 (2013). 
17. L. Chen, M. Subirade, Eur. J. Pharm. Biopharm. 65(3), 354-362 (2007).

18. S.M. Hosseini, H. Hosseini, M.A. Mohammadifar, J.B. German, A.M. Mortazavian, A.

Mohammadi, K. Khosravi-Darani, S. Shojaee-Aliabadi, R. Khaksar, Carbohydr. Polym. 103, 573580 (2014).

19. E. Hasanvand, M. Fathi, A. Bassiri, M. Javanmard, R. Abbaszadeh, Novel starch based nanocarrier for vitamin D fortification of milk: Production and characterization. Food Bioprod. Process. 96, 264-277 (2015).

20. E.I. Paramera, S.J. Konteles, V.T. Karathanos, Food Chem. 125(3), 913-922 (2011).

21. L. Zhao, F. Temelli, L. Chen, J. Funct. Foods 34, 159-167 (2017).

22. C.J. Porter, N.L. Trevaskis, W.N. Charman, Nat. Rev. Drug Discov. 6(3), 231-248 (2007).

23. L.M. Ward, I. Gaboury, M. Ladhani, S. Zlotkin, Can. Med. Assoc. J. 177(2), 161-166 (2007).

24. B. Ozturk, S. Argin, M. Ozilgen, D.J. McClements, Food Chem. 187, 499-506 (2015)

25. R.R. Eitenmiller, W. Landen Jr, L. Ye, For the Health and Food Sciences, 2nd edn. (Boca Raton, 2016), pp. 83-112.

26. B. Delavari, A.A. Saboury, M.S. Atri, A. Ghasemi, B. Bigdeli, A. Khammari, P. Maghami, A.A. Moosavi-Movahedi, T. Haertlé, B. Goliaei, Food Hydrocoll. 45, 124-131 (2015).

27. J.N. Pedersen, H.S. Frislev, J.S. Pedersen, D.E. Otzen, J. Dairy Sci. 99(10), 7755-7767 (2016).

28. O. Paucar, F. Tulini, M. Thomazini, J. Balieiro, E. Pallone, C. Favaro-Trindade, Food Bioprod. Process. 100, 344-350 (2016).

29. H. Belitz, W. Grosch, P. Schieberle, Food Chem, 4nd edn. (Springer-Verlag, Berlin Heidelberg, 2009), p. 406.

30. M.F. Holick, Vitamin D deficiency. N Engl J Med. 357(3), 266-281 (2007).

31. M.R. Patel, S. Martin-Gonzalez, M. Fernanda, J. Food Sci. 77(1), 8-13 (2012). 